\title{
POINTWISE CONVERGENCE AND SEMIGROUPS ACTING ON VECTOR-VALUED FUNCTIONS
}

\author{
MICHAEL G. COWLING ${ }^{凶}$ and MICHAEL LEINERT
}

(Received 30 September 2010)

\begin{abstract}
A submarkovian $C_{0}$ semigroup $\left(T_{t}\right)_{t \in \mathbb{R}^{+}}$acting on the scale of complex-valued functions $L^{p}(X, \mathbb{C})$ extends to a semigroup of operators on the scale of vector-valued function spaces $L^{p}(X, E)$, when $E$ is a Banach space. It is known that, if $f \in L^{p}(X, \mathbb{C})$, where $1<p<\infty$, then $T_{t} f \rightarrow f$ pointwise almost everywhere. We show that the same holds when $f \in L^{p}(X, E)$.
\end{abstract}

2010 Mathematics subject classification: primary 47D06; secondary 7D07, 46G20.

Keywords and phrases: semigroups, vector-valued, pointwise convergence.

\section{Introduction}

Let Lebesgue space $L^{2}(X, \mathbb{C})$, satisfying the following conditions:

(1) $\left\|T_{t} f\right\|_{p} \leq\|f\|_{p}$, for all $t \in \mathbb{R}^{+}$for all $f \in L^{p} \cap L^{2}(X, \mathbb{C})$ for all $p \in[1, \infty]$;

(2) $T_{t}=T_{t}^{*}$, for all $t \in \mathbb{R}^{+}$;

(3) $\lim _{t \rightarrow 0+}\left\|T_{t} f-f\right\|_{p}=0$, for all $f \in L^{p} \cap L^{2}(X, \mathbb{C})$ for all $p \in[1, \infty)$;

(4) $T_{t} f \in L^{2}(X, \mathbb{C})^{+}$, for all $t \in \mathbb{R}^{+}$for all $f \in L^{2}(X, \mathbb{C})^{+}$;

(5) $\int_{X} T_{t} f d \mu=\int_{X} f d \mu$, for all $t \in \mathbb{R}^{+}$for all $f \in L^{1} \cap L^{2}(X, \mathbb{C})$.

We say that $\left(T_{t}\right)_{t \in \mathbb{R}^{+}}$is a markovian $C_{0}$ semigroup. Clearly, $\left(T_{t}\right)_{t \in \mathbb{R}^{+}}$extends uniquely by continuity to a $C_{0}$ semigroup on each space $L^{p}(X, \mathbb{C})$ when $1 \leq p<\infty$. We shall abuse notation and write $T_{t}$ for the extension of $T_{t}$ to $L^{p}(X, \mathbb{C})$.

If $\left(T_{t}\right)_{t \in \mathbb{R}^{+}}$satisfies only conditions (1)-(3), then we say that $\left(T_{t}\right)_{t \in \mathbb{R}^{+}}$is submarkovian. This nomenclature is at least in part justified by the following result of Taggart [5].

THEOREM 1.1. Suppose that $\left(T_{t}\right)_{t \in \mathbb{R}^{+}}$is a submarkovian $C_{0}$ semigroup. Then there exists a $C_{0}$ semigroup of operators $\left(\tilde{T}_{t}\right)_{t \in \mathbb{R}^{+}}$on $L^{2}(X, \mathbb{C})$ satisfying conditions $(1)-(4)$

It is a pleasure for the first-named author to acknowledge the generous support of an Alexander von Humboldt Foundation Research Prize, and the hospitality of the University of Heidelberg.

(C) 2011 Australian Mathematical Publishing Association Inc. 0004-9727/2011 \$16.00 
above, and the additional condition

$$
\left|T_{t} f\right| \leq \tilde{T}_{t}|f| \quad \forall f \in L^{2}(X, \mathbb{C}) \forall t \in \mathbb{R}^{+} .
$$

One of the keys to this theorem is that conditions (1)-(3) imply the existence of a locally integrable kernel $k_{t}: X \times X \rightarrow \mathbb{C}$ such that

$$
T_{t} f(x)=\int_{X} k_{t}(x, y) f(y) d \mu(y) \quad \forall t \in \mathbb{R}^{+} \forall f \in L^{2}(X, \mathbb{C}) ;
$$

further,

$$
\begin{aligned}
& \underset{x \in X}{\operatorname{ess} \sup _{X}} \int_{X}\left|k_{t}(x, y)\right| d \mu(y) \leq 1, \\
& \underset{y \in X}{\operatorname{ess} \sup } \int_{X}\left|k_{t}(x, y)\right| d \mu(x) \leq 1,
\end{aligned}
$$

and $k_{t}(x, y)=\bar{k}_{t}(y, x)$ for almost all $(x, y)$ in $X \times X$. These conditions show that $\left(T_{t}\right)_{t \in \mathbb{R}^{+}}$extends uniquely by weak-star continuity to a weak-star continuous semigroup on $L^{\infty}(X, \mathbb{C})$.

The semigroup $\left(\tilde{T}_{t}\right)_{t \in \mathbb{R}^{+}}$constructed by Taggart, which also satisfies conditions (1)-(3), has a family of associated kernels, $\tilde{k}_{t}$ say, which satisfy $\left|k_{t}\right| \leq \tilde{k}_{t}$ on $X \times X$.

Stein [4] studied markovian semigroups. Inter alia, he showed that if $1<p<\infty$ and $f \in L^{p}(X, \mathbb{C})$, then $T_{t} f \rightarrow f$ pointwise almost everywhere as $t \rightarrow 0+$. Later, Cowling [1] relaxed the hypotheses in order to deal with submarkovian semigroups. It is perhaps worth pointing out exactly what this pointwise convergence means, since limits on uncountable sets can be somewhat problematic in Lebesgue spaces. Because we will come across questions where the distinction between functions and equivalence classes of functions is important, we distinguish between the space $\mathcal{L}^{p}(X, \mathbb{C})$ of measurable complex-valued functions, defined almost everywhere on $X$, such that $\|f\|_{p}<\infty$, where

$$
\|f\|_{p}=\left(\int_{X}|f(x)|^{p} d \mu(x)\right)^{1 / p},
$$

and the space $L^{p}(X, \mathbb{C})$ of equivalence classes of such functions, modulo equality almost everywhere.

As Stein observed, if $1<p<\infty$, then the $C_{0}$ semigroup $\left(T_{t}\right)_{t \in \mathbb{R}^{+}}$on $L^{p}(X, \mathbb{C})$ extends analytically to a semigroup $\left(T_{z}\right)_{z \in \Gamma_{\theta}}$ on $L^{p}(X, \mathbb{C})$, where

$$
\theta=(1 / 2-|1 / p-1 / 2|) \pi
$$

and $\Gamma_{\theta}$ is the cone $\{z \in \mathbb{C}:|\arg (z)|<\theta\}$. This angle is not best possible: see Liskevich and Perelmuter [3]. This cone is a countable union of disks. In each disk $D\left(z_{j}, r_{j}\right)$ with centre $z_{j}$ and radius $r_{j}$, we may write $T_{z} f$ as a convergent series in powers 
of $z-z_{j}$, with coefficients in $L^{p}(X, \mathbb{C})$. By choosing representatives from the equivalence class of each of the coefficients, we obtain a series $S_{j}(x, z)$, given by

$$
S_{j}(x, z)=\sum_{n \in \mathbb{N}} f_{j, n}(x)\left(z-z_{j}\right)^{n} .
$$

The series $S_{j}(x, \cdot)$ converges for almost all $x \in X$ to an analytic function in $D\left(z_{j}, r_{j}\right)$, and $S(\cdot, z)$ exists almost everywhere and lies in $\mathcal{L}^{p}(X, \mathbb{C})$ for all $z \in D\left(z_{j}, r_{j}\right)$. By making such a choice of representatives in each disk, we can arrange that $z \mapsto T_{z} f(x)$ is analytic in $\Gamma_{\theta}$ for almost all $x \in X$, and so we can consider expressions such as $\lim _{t \rightarrow 0+} T_{t} f(x)$; they are the same as limits where $t$ is restricted to be rational.

As argued by Taggart [5], if $E$ is a separable complex Banach space, then each operator $T_{t}$ on $L^{p}(X, \mathbb{C})$ extends to an operator $T_{t} \otimes I$ on $L^{p}(X, E)$, the space of equivalence classes of measurable $E$-valued functions $F$ such that $\|F\|_{E, p}<\infty$, where

$$
\|F\|_{E, p}=\left(\int_{X}\|F(x)\|_{E}^{p} d \mu(x)\right)^{1 / p} .
$$

In particular, if $F=\sum_{j=1}^{n} f_{j} \xi_{j}$, where $f_{j} \in L^{p}(X, \mathbb{C})$ and $\xi_{j} \in E$ for each $j$, then

$$
\left(T_{t} \otimes I\right) F=\sum_{j=1}^{n} T_{t} f_{j} \xi_{j}
$$

Alternatively, we may write

$$
\left(T_{t} \otimes I\right) F(x)=\int_{X} k_{t}(x, y) F(y) d \mu(y) .
$$

By using this representation, we may show that

$$
\left\|\left(T_{t} \otimes I\right) F\right\|_{E} \leq \int_{X} \tilde{k}_{t}(\cdot, y)\|F(y)\|_{E} d \mu(y)=\tilde{T}_{t}\|F\|_{E} .
$$

Further, for all $p \in[1, \infty)$, the semigroup $\left(T_{t} \otimes I\right)_{t \in \mathbb{R}^{+}}$on $L^{p}(X, E)$ satisfies the following conditions:

(1v) $\left\|\left(T_{t} \otimes I\right) F\right\|_{p} \leq\|F\|_{p}$, for all $t \in \mathbb{R}^{+} \forall F \in L^{p} \cap L^{2}(X, E)$;

(3v) $\lim _{t \rightarrow 0+}\left\|T_{t} F-F\right\|_{p}=0$, for all $F \in L^{p} \cap L^{2}(X, E)$.

Of course, it no longer makes sense to suggest that $T_{t} \otimes I$ is self-adjoint (unless $E$ is a Hilbert space). It is a reasonable question to ask whether $\left(T_{t} \otimes I\right) F$ converges to $F$ pointwise almost everywhere as $t \rightarrow 0+$. In [5], this is shown subject to some additional hypotheses on $E$. The point of this note is that $E$ can be any separable Banach space.

THEOREM 1.2. Suppose that $\left(T_{t}\right)_{t \in \mathbb{R}^{+}}$is a submarkovian $C_{0}$ semigroup, that $E$ is a separable Banach space, and that $1<p<\infty$. Then for all $F$ in $L^{p}(X, E)$,

$$
\lim _{t \rightarrow 0+}\left(T_{t} \otimes I\right) F=F
$$

pointwise almost everywhere in $X$. 
There has been interest in vector-valued semigroups in the context of maximal regularity. See [2] for more information and bibliography.

\section{Proof}

Take $F \in \mathcal{L}^{p}(X, E)$. We consider the $[0, \infty]$-valued function

$$
x \mapsto \limsup _{t \rightarrow 0+}\left\|\left(T_{t} \otimes I\right) F(x)-F(x)\right\|_{E} .
$$

A priori, we do not know that this function is measurable. However, we do know that, given $\epsilon$ in $\mathbb{R}^{+}$, we may write $F$ as $F_{\otimes}+F_{\epsilon}$, where $F_{\otimes}$ is a finite tensor product function, that is,

$$
F_{\otimes}(x)=\sum_{j=1}^{n} f_{j}(x) \xi_{j},
$$

where $f_{j} \in \mathcal{L}^{p}(X, \mathbb{C})$ and $\xi_{j} \in E$ for each $j$, and $\left\|F_{\epsilon}\right\|_{p}<\epsilon$. By the pointwise convergence result for the scalar case,

$$
\limsup _{t \rightarrow 0+}\left\|\left(T_{t} \otimes I\right) F_{\otimes}(x)-F_{\otimes}(x)\right\|_{E}=0
$$

for almost all $x \in X$. Further,

$$
\left\|\left(T_{t} \otimes I\right) F_{\epsilon}-F_{\epsilon}\right\|_{E} \leq\left\|\left(T_{t} \otimes I\right) F_{\epsilon}\right\|_{E}+\left\|F_{\epsilon}\right\|_{E} \leq \tilde{T}_{t}\left\|F_{\epsilon}\right\|_{E}+\left\|F_{\epsilon}\right\|_{E},
$$

where $\left(\tilde{T}_{t}\right)_{t \in \mathbb{R}^{+}}$is as in Theorem 1 , and, as proved in [1, 4], it is natural and possible to choose the representatives of the real-valued functions $\tilde{T}_{t}\left\|F_{\epsilon}\right\|_{E}$ such that $\tilde{T}^{*}\left\|F_{\epsilon}\right\|_{E}$, defined by

$$
\tilde{T}^{*}\left\|F_{\epsilon}\right\|_{E}=\sup _{t \in \mathbb{R}^{+}} \tilde{T}_{t}\left\|F_{\epsilon}\right\|_{E},
$$

is well defined and measurable, and satisfies

$$
\left\|\left(\tilde{T}^{*}\left\|F_{\epsilon}\right\|_{E}\right)\right\|_{p} \leq C_{p}\|\| F_{\epsilon}(\cdot)\left\|_{E}\right\|_{p}=C_{p}\left\|F_{\epsilon}\right\|_{E, p}<C_{p} \epsilon .
$$

It follows that, by choosing suitable representatives for each $\left(T_{t} \otimes I\right) F$,

$$
\limsup _{t \rightarrow 0+}\left\|\left(T_{t} \otimes I\right) F(\cdot)-F(\cdot)\right\|_{E}
$$

is equal to 0 outside a set of measure 0 , and hence we have pointwise convergence almost everywhere.

One can be more explicit about the sets of measure 0 that occur. We can write $F$ as a convergent sum $\sum_{j \in \mathbb{N}} F_{j}$, where each $F_{j}$ is a finite tensor. We can then choose representatives so that each $\left(T_{t} \otimes I\right) F_{j}(\cdot)$ is continuous in $t$, and $\left(T_{t} \otimes I\right) F_{j} \rightarrow F_{j}$ outside a null set $N_{j}$. When all is said and done, $\left(T_{t} \otimes I\right) F$ converges pointwise to $F$ outside $\bigcup_{j \in \mathbb{N}} N_{j}$. 


\section{Acknowledgement}

It is a pleasure to thank Werner Ricker for his useful comments on this paper.

\section{References}

[1] M. G. Cowling, 'Harmonic analysis on semigroups', Ann. of Math. (2) 117 (1983), 267-283.

[2] P. C. Kunstmann and L. Weis, 'Maximal $L^{p}$-regularity for parabolic equations, Fourier multiplier theorems and $H^{\infty}$-functional calculus', in: Functional Analytic Methods for Evolution Equations, Lecture Notes in Mathematics, 1855 (Springer, Berlin, 2004), pp. 65-311.

[3] V. A. Liskevich and M. A. Perelmuter, 'Analyticity of submarkovian semigroups', Proc. Amer. Math. Soc. 123 (1995), 1097-1104.

[4] E. M. Stein, Topics in Harmonic Analysis Related to the Littlewood-Paley Theory (Princeton University Press, Princeton, NJ, 1970).

[5] R. J. Taggart, 'Pointwise convergence for semigroups in vector-valued $L^{p}$ spaces', Math. Z. 261 (2009), 933-949.

MICHAEL G. COWLING, School of Mathematics and Statistics, University of New South Wales, UNSW Sydney 2052, Australia e-mail: m.cowling@unsw.edu.au

MICHAEL LEINERT, Institut für angewandte Mathematik, Im Neuenheimer Feld 294, Ruprechts-Karl-Universität Heidelberg, D-69120 Heidelberg, Germany e-mail: leinert@math.uni-heidelberg.de 